Diánoia, vol. 11, no. 11, 1965

\title{
ALGUNAS CONCEPCIONES ERRÓNEAS SOBRE LÓGICA MODERNA
}

Acerca de la lógica moderna están difundidas una multitud de concepciones erróneas. Sólo en forma ocasional se encuentran afirmaciones explícitamente claras que reflejan esos malentendidos, pero quienquiera que haya examinado algo más que superficialmente la lógica moderna y que la haya discutido con estudiantes, colegas u otras personas competentes, reconocerá que algunas opiniones y actitudes equivocadas respecto de la lógica son muy comunes.

Además, estas concepciones erróneas parecen impedir el uso de la lógica moderna como un instrumento en las discusiones intelectuales. Los lógicos no pretenden que ésta sea la única herramienta requerida; tampoco suponen o propugnan que debiera ser aplicada a todas las variedades de discurso. Pero su uso más extenso puede mejorar muchas discusiones y en tanto que las concepciones erróneas acerca de ella estorban su uso más amplio, pueden ser perjudiciales a la vida intelectual entera de nuestros tiempos.

Por "lógica moderna" se significa "lógica deductiva moderna". El término "Iógica simbólica" es también usado frecuentemente como sinónimo de "lógica deductiva moderna", mas el primer término parece alimentar un malentendido que se discutirá posteriormente, y aquí será útil decir "lógica moderna". También, para los presentes designios, podemos limitar la referencia del término a las porciones elementales que están relacionadas con argumentos cuyas formas lógicas implican esencialmente sólo "no", "y", "o", "si", "todos", "algunos", y sinónimos de estas expresiones (o convenientes duplicados notacionales de estos conceptos).

Una concepción errónea perjudicial es que la lógica moderna representa una desviación radical de la lógica vieja o "tradicional"; ${ }^{1}$ que las dos tienen poco en común y que son alternativas tan fuertemente opuestas como, digamos, Budismo y Cristianismo, o Comunismo y Democracia. Pero el contenido de la vieja lógica y la teoría moderna no son mutuamente excluyentes, la mayor parte de los argumentos considerados como válidos en el siglo xix todavía son considerados como argumentos válidos. Históricamente, toda la

1 Por "lógica tradicional" se significa aqui, grosso modo, el trabajo típico del siglo diecinueve, que es presentado en recientes libros de texto elementales como "lógica tradicionàl". El término es también usado para referirse a la tradición escolástica en lógica, pero este artículo no se refiere a ese desarrollo. Se requeriria una discusión mucho más detallada para asegurar una confrontación o diálogo genuinos, entre lógica escolástica y lógica moderna. 
lógica deductiva se ha ocupado en aislar, estudiar y sistematizar aquellos modelos o formas de inferencia, que invariablemente conducen a conclusiones verdaderas cuando las premisas son verdaderas. Esto es lo que la lógica tradicional hacía y lo que la lógica moderna hace. ¿Cuál es, pues, la diferencia entre las dos?

La diferencia principal es que la lógica moderna ha expandido enormemente la esfera de las formas argumentativas cuya validez puede ser determinada. Los viejos principios constituyen sólo un pequeño fragmento del cuerpo de la teoría moderna; los más poderosos métodos ahora disponibles permiten aplicaciones a muchos argumentos que estaban más allá de las capacidades de las viejas técnicas. A manera de ejemplo, consideremos este argumento:

Cualquier uso de una expresión es un significado de esta expresión.

Por lo menos algunas expresiones tienen un uso.

Por consiguiente, existen significados para algunas expresiones.

La lógica tradicional no nos proporciona medios para mostrar que este argumento es válido; la teoría de la cuantificación lo establece fácilmente. ${ }^{2}$ Quien limite sus principios lógicos al viejo organon actúa como un granjero que prefiere labrar sus millares de acres con un arado de madera y un par de mulas en vez de usar un tractor y un arado de reja múltiple.

Una segunda diferencia entre la vieja y la nueva lógica reside en la eliminación de varias insuficiencias en aquellas partes de la lógica que caen dentro de la competencia de la primera. Aunque estas dificultades son bien conocidas, se citará un ejemplo para ilustrar el punto. De acuerdo con la vieja teoría, una proposición del tipo A (de la forma 'Todo $S$ es $P$ ') implica su contrapositiva ('Todo no-P es no-S'), que es otra proposición del tipo A. Una proposición del tipo A también implica la correspondiente proposición del tipo I ('Algún $S$ es $P$ '). Así, 'Todos los hombres son idénticos a sí mismos' implica 'Todas las cosas no idénticas a sí mismas son nohombres'. Ésta, a su vez, implica "Algunas cosas no idénticas a sí mismas son no-hombres". Pero empezamos con una proposición verdadera y concluimos con una falsa. (La conclusión del tipo I es falsa porque no existe nada que sea no-idéntico a sí mismo, sea hombre o no). Los principios de la vieja lógica constituyen un sistema inconsistente y sin duda está fuera de controversia que una lógica inconsistente es inadecuada hasta que se la enmiende. Los sistemas modernos de lógica proporcionan un comprobable tratamiento consistente de tales cuestiones y, 'de este modo, asi como por su mayor poder, representa un avance sobre su predecesora.

Pero más que las diferencias, la continuidad de la nueva con la vieja

$2 \mathrm{El}$ argumento en sł mismo es de interés, desde que los seguidores de Wittgenstein parecen aceptar las premisas; pero algunos, por ejemplo el profesor Ryle, niegan la conclusión. 
necesita ser más acentuada. Aun cuando algunos de los principios de la lógica tradicional son rechazados en la teoría moderna con objeto de asegurar consistencia, es necesario acentuar que la mayoria de los principios, que llegaron a través de los siglos, han sido conservados. Este punto puede ser obscurecido si uno compara superficialmente un par de libros que representen los dos puntos de vista. Un texto tradicional dedica usualmente un gran número de páginas a diversos tipos de inferencia -el silogismo disyuntivo, por ejemplo- que son mencionados sólo brevemente o en manera alguna en un libro como Métodos de la Lógica de Quine. Uno podría suponer que existe una diferencia radical en el contenido entre los dos, o que hay poco en común entre ellos. Frecuentemente los escritores sobre lógica moderna guardan silencio o discuten sólo brevemente tópicos que recibieron un extenso tratamiento en el pasado. Pero esto se explica fácilmente, porque los tópicos están generalmente subsumidos bajo principios nuevos que son más generales, más poderosos y más ampliamente aplicables. Los principios tradicionales son usualmente casos especiales de los nuevos. Suponer que los nuevos y los viejos difieren substancialmente o que son alternativas entre las cuales cada quien hace su elección, es malentender la naturaleza de la lógica moderna.

Una segunda concepción errónea parece ser fomentada por el uso del término "lógica simbólica". El término es frecuentemente usado en una forma que sugiere que la vieja lógica no usó un simbolismo y que la lógica moderna, por usar una representación simbólica de los argumentos - cualquier representación simbólica - representa una desviación radical de la tradición. Como algunas veces se usa, el término "lógica simbólica" lleva consigo una sobreacentuación de lo esotérico, lo artificial y de inaplicabilidad a casos concretos. Ya se ha dicho bastante acerca de la continuidad de la teoría tradicional y moderna y de la tremenda aplicabilidad de la última, pero el nuevo simbolismo merece algún comentario.

Como se mencionó al principio, todas las variedades de la lógica deductivas se relacionan con las formas de la argumentación válida. La teoría de estas formas puede ser desarrollada empleando descripciones verbales de ellas; por ejemplo, las dos premisas en un caso dado pueden ser descritas como una proposición hipotética y el antecedente de esta hipotética. Pero estas descripciones, cuando las formas no son sumamente simples, se volverían tan complejas que los principios de la inferencia válida, enunciados por su medio, se volverían incomprensibles. Es mucho más conveniente -y mucho más gráfico- usar algún simbolismo para representar las formas de argumento.

El punto es tan viejo como los escritos lógicos de Aristóteles. En ver. dad, precisamente porque él usó un simbolismo en el que las letras del alfabeto griego representaban términos, por eso es considerado como el fun- 
dador de la lógica en Occidente. Desde sus comienzos, la lógica ha sido simbólica. Si en general hemos de tener una teoría de la deducción, necesita estar relacionada con modelos o formas de inferencia, y si hemos de discutir estas formas clara y convenientemente, tenemos que usar algún simbolismo. No hay nada equivocado o misterioso respecto del uso del simbolismo en lógica. Pero una objeción a cualquier cosa simbólica en lógica es a la vez equivocada $y$, al menos, un poco misteriosa en el sentido en que sería misterioso que un hombre cuerdo se amputase su brazo derecho sano.

La única cuestión seria referente al simbolismo es qué sistema de símbolos debiera usarse. La vieja teoría adoptó: “Todo $S$ es $\mathrm{P}$ ”, como la forma de una variedad de proposición muy frecuentemente usada; en desarrollos más recientes la forma de estas proposiciones es tomada así: "Para todo objeto X, si X es S, entonces X es $P$." Existen dos alternativas: ¿cuál debería ser elegida? La respuesta está íntimamente conectada con lo que se dijo al principio respecto a la habilidad de la lógica moderna para tratar la cuestión de la validez de muchas clases de argumentos que se hallan fuera de la competencia de la vieja doctrina. Este aumento de poder o aplicabilidad se ha efectuado a través del desarrollo del nuevo simbolismo que permite la identificación de detalles relevantes de las estructuras lógicas de las proposiciones - detalles no ofrecidos en las formas tradicionales semejantes a la citada. (El ejemplo citado del nuevo simbolismo, por supuesto no ofrece ningún detalle mayor que la forma tradicional, pero es sólo un simple elemento de un sistema de símbolos, una gran parte de los cuales no tiene equivalente en la lógica tradicional.)

Si uno entiende estas cuestiones correctamente, reconocerá que toda la lógica deductiva es formal, que cierto simbolismo es el único modo conveniente de tratar con formas, que la lógica tradicional fue justamente tan simbólica como la lógica moderna y que el nuevo simbolismo es preferible al viejo porque facilita el tránsito a una teoría más poderosa.

Pero tal vez el simbolismo sea la fuente de una tercera concepción errónea, a saber, que la lógica moderna es absurdamente compleja y que es el producto de una ingenuidad más bien loca o de una mente fascinada por construcciones elaboradas. Dentro del cuerpo de la lógica, que es el asunto de este artículo, hay complejidad, pero hay también porciones de considerable simplicidad - de hecho, una simplicidad tal que estas porciones han sido enseñadas con éxito a niños de la escuela elemental. $Y$ donde existe complejidad, no es complejidad por la complejidad misma, sino complejidad por un propósito serio. Pues la lógica tiene su base en el lenguaje que todos usamos- un lenguaje que es enormemente sutil y complejo. Algunas inferencias comunes y aparentemente simples, cuando se analizan, se ven convertirse en formas de considerable complejidad y únicamente podemos atenernos a las partes más simples de la lógica, sacrificando mucho de lo que 
queremos hacer. El lenguaje en que discutimos problemas intelectuales -incluyendo los de la filosoffa- a menudo es de una naturaleza lógicamente compleja y el lógico, aun cuando a menudo es muy hábil, se ocupa del aislamiento, estudio y prueba de las inferencias deductivas involucradas. En tanto estas inferencias son simples, bastarán técnicas lógicas simples; en tanto son complejas, se requerirán técnicas más complicadas.

Una cuarta concepción errónea es el parecer de que la lógica moderna se ocupa solamente en la manipulación de símbolos o en el "malabarismo de símbolos". Es verdad, por supuesto, que en la lógica moderna algún trabajo se halla en el campo de la sintaxis, y este campo incluye reglas que permiten operaciones con las formas simbólicas de los argumentos. Pruebas y derivaciones, por ejemplo, son construidas de acuerdo con reglas que permiten transformaciones de cadenas de símbolos en otras cadenas de símbolos, pero estas transformaciones no son el único interés de la lógica, ni las reglas se formulan para convertirlas en algo sin objeto. Unos cuantos comentarios acerca de las operaciones con símbolos en la lógica moderna puede ser útil para entender su raison d'etre.

Se acentuó al principio que la lógica moderna tiene aplicaciones extensas. Generalmente, la aplicación de principios lógicos a argumentos verbales (en cualquier campo) ímplica cuatro pasos. El primero es el comparativamente asistemático, y algunas veces difícil, de transformar el argumento en otro argumento cuya forma lógica sea transparente. El segundo paso es la representación del argumento en una forma simbólica. En el tercer paso es determinada la validez de la forma, y finalmente (en casos apropiados) la validez del argumento original es inferida de la validez de la forma. Por supuesto, en el tercero de estos cuatro pasos es donde se efectúan las operaciones sintácticas. La teoria lógica, en un sentido estricto del término, no se refiere al primero, segundo o cuarto pasos, y únicamente por ignorar el contexto de aplicaciones para las que ha sido desarrollada la teoría es por lo que puede malentenderse la teoría como no referida a otra cosa que al malabarismo de signos.

El uso de manipulaciones simbólicas en el trabajo lógico moderno, sin embargo, es uno de los contrastes entre éste y el cuerpo de la vieja doctrina, y una comprensión de la necesidad de estas reglas ayudará a evitar concepciones erróneas. Más específicamente, el contraste es entre una teoría que en gran medida tiene el carácter de una lista o compendio de formas válidas simples y una teoría que mediante reglas de transformación prepara la construcción de deducciones (o "derivaciones" o "pruebas"). En el caso anterior se muestra que un argumento es válido mostrando que tiene una de las formas normales reconocidas, o mostrando que tiene una forma que satisface un conjunto de reglas tales como las del silogismo. Por otra parte, las deducciones construidas de acuerdo con reglas establecen la validez de 
los argumentos con la introducción de pasos intermedios, a través de los cuales se asegura la conexión de las premisas con la conclusión.

Pero, ¿por qué debería haber este cambio de un catálogo a una técnica de operaciones con formas, especialmente cuando que el uso de la última requiere algunas veces tiempo e ingenio? La respuesta se halla íntimamente conectada con lo que se dijo al principio respecto a la esfera más amplia de aplicabilidad de la lógica moderna y del enorme auge en el número de formas de argumento cuya validez puede ser determinada. Cuando - el número de formas discutidas es relativamente pequeño, éstas pueden ser enlistadas y los argumentos verificados con dicha lista. En los Elementos de Lógica (1857) del arzobispo Whatley, por ejemplo, hay una sección titulada "Compendio Sintético", en la cual (calculando las diversas variedades del silogismo) se describen cerca de treinta formas válidas. La lógica moderna, por el contrario, reconoce como válido un número ilimitado de modelos.

Aunque muchos textos modernos enlistan un número de los que son usados con gran frecuencia, es imposible enlistarlos a todos, y cualquier compilación muy larga de formas escogidas sería engorrosa e ineficaz. Es necesario recurrir a otros artificios para probar las formas de validez, y es característico de la lógica moderna ofrecer métodos generales para probar clases completas de formas de argumento. En estos métodos generales es donde existen operaciones con símbolos.

Una comprensión de las manipulaciones simbólicas en la lógica moderna implica también un segundo punto. La teoría no consiste solamente en reglas para operar con símbolos. Es esencial justificar las reglas mediante una teoría semántica que garantice que las operaciones nunca permitirán una inferencia de una verdad a una falsedad. El "malabarismo de signos" no se hace arbitrariamente; cualquier regla en uso ha sido justificada como segura y esta justificación está siempre en el fondo cuando se hacen las aplicaciones. Visto superficialmente, sin una comprensión de la semántica que lo justifica, el uso de reglas puede parecer arbitrario, fútil y misterioso; pero cuando se cae en la cuenta de que lo racional de las reglas está en el hecho de que establecen un gran número de inferencias válidas, al mismo tiempo que excluyen las inválidas, y de que este aspecto suyo ha sido comprobado, queda poca razón para objetar las manipulaciones simbólicas.

Aunque se ha trazado el contraste entre un compendio de formas válidas y un sistema de reglas para la manipulación de signos, la vieja lógica proporciona de hecho reglas para el "malabarismo" de símbolos. Frecuentemente se dice en los viejos textos que una forma de argumento es válida y se dice también que la forma de conclusión puede ser inferida de formas de premisa. Enunciados acerca de la validez de formas y reglas que permiten manipulaciones simbólicas a menudo parecen ser considerados como 
sinónimos. Whatley $(1857$, p. 69$)$ designa la siguiente forma como el "silogismo condicional constructivo":

Si A es B, C es D; pero A es B, por tanto C es D. Él consideró este modelo de argumento como válido, pero también enunció como "la regla primera" (la cursiva es mía) en su tratamiento de las "condicionales": "concedido el antecedente, el consecuente puede ser inferido". $Y$ hay un argumento semántico para justificar la regla de operación con formas. Básicamente, entonces, el uso de reglas semánticamente justificadas para manipular formas no es una novedad introducida por los lógicos modernos. Nosotros tenemos ahora una variedad más amplia de reglas, las formas involucradas son diferentes y la complejidad de las posibles manipulacionès ha aumentado, pero las razones sobre estos aspectos de la lógica moderna han sido suficientemente discutidas en las primeras partes de este artículo.

Una quinta concepción errónea -más bien ingenua, pero sin embargo muy difundida - es la confusión de la lógica moderna sea con el positivismo lógico, sea con una o con todas las diversas escuelas de filosofía lingüística. El criterio de verificabilidad (o "empírico") de la significatividad, obviamente, no es un principio en ningún sistema normal de lógica moderna. La única conexión entre lógica y positivismo (diversa de algunos insignificantes azares históricos, tal como el de que Carnap sea a la vez lógico y positivista) es que en las formulaciones del principio de verificabilidad empleó algunas nociones de lógica tales como la de implicación. Otro tanto, sin embargo, puede decirse respecto de una gran parte de la filosofía. Kant, Hume y la mayoría de los filósofos de la historia usaron también nociones de la lógica de su tiempo, pero esto sólo no los hace lógicos. Los escritos de los positivistas a menudo intentaron usar la lógica, pero sus escritos filosóficos, obviamente, no son trabajos que presenten una teoría sistemática en lógica. Lenguaje, Verdad y Lógica no es, en ningún sentido, un tratado en el campo de la lógica moderna, y ni el criterio empirista de significatividad, ni ninguna teorfa emotiva de cualquier cosa es un principio que deba encontrarse en cualquier tratado normal. La lógica moderna y el empirismo lógico son independientes por completo la una del otro, y ninguno que desee hacer uso de la primera, está, en modo alguno, comprometido con el último.

Casi lo mismo puede decirse respecto de las escuelas clasificadas de filosofía lingüística. En particular, debería acentuarse que en ningún sistema de lógica moderna es ni un axioma ni un teorema que la lógica o el análisis lingüístico sea la única tarea de la filosofía. El que no es lógico ha intentado siempre mostrar que tal principio es válido (en el sentido estricto de "válido" que se ha empleado a través de este artículo). Algunos lógicos -especialmente aquellos que son también matemáticos- raramente o nunca discuten la naturaleza de la filosofía u otras cuestiones filosóficas. 
Otros lógicos se ocupan en la filosofía, pero el punto importante es éste: en la lógica moderna misma no hay nada que prohiba la discusión de cualquier problema, sea el que sea, metafísico, ético, estético u otro. En vez de oponerse a la actividad filosófica, el uso más extenso de la lógica puede frecuentemente facilitarla.

Una última vaga concepción errónea parece ver a la lógica moderna como inhumanamente constrictiva; se piensa que el rigor y la exactitud resultan al reprimir el indomable espíritu del hombre y que la lógica se contrapone a las actividades creadoras e imaginativas de los seres humanos. Varios puntos merecen comentarse.

Primero, puede notarse que esta actitud (no inhabitual en personas de propensión a la vez literaria y anticientifica) se dirige tanto hacia la vieja lógica como hacia la lógica moderna. Tiende a ser una oposición a toda ló gica -de hecho, casi a cualquier tipo de disciplina intelectual.

En segundo lugar, no hay en ninguna teoría lógica - vieja o nuevanada que prohiba cualquier clase de libre especulación o actividad artística, sea la que sea. Ningún tratado sobre la deducción contiene ley alguna o regla que ponga en entredicho la expresión de las energías creadoras de cualquiera. Es legítimo criticar a un escritor por no haberse servido él mismo de las técnicas que lo habrian ayudado a lograr su ostensible propósito. Pero aun aquí el principio (burdamente, que la lógica debería ser usada donde pueda ayudar) es un principio cuasi-ético, y no un principio dentro de un sistema lógico. La lógica en sí misma no dice que la lógica debería ser usada.

En tercer lugar, la presente concepción errónea parece ver el uso de la lógica en que produjera sólo resultados negativos - como si produjera sólo rechazos de percepciones de importancia. Esto está lejos del caso real, pero en cuanto el criticismo lógico muestra algunas veces que creencias estimadas son insostenibles, no es antitético a las aspiraciones humanas. Pues el criticismo lógico, en general, indicará lo inadecuado de una opinión solamente señalando su incompatibilidad con otras apreciadas doctrinas. Y si uno busca la verdad - sea por satisfacción intelectual o por designios más "prácticos"-, sus aspiraciones obviamente se aproximarán más a la realización, a medida que resuelva las inconsistencias (si hay algunas) que infectan su pensamiento. Cuando los resultados de las críticas lógicas son negativos, contribuyen a la realización de los propósitos humanos.

Pero la lógica deductiva también tiene sus aspectos positivos, pues el descubrimiento de las implicaciones de una teoría, frecuentemente, en un sentido, es descubrimiento de una nueva teoría, y proporciona una inteligencia más comprensiva de la teoría original misma. Y como contribución más específica de la lógica está la de la construcción de modelos, los cuales son de creciente importancia, no sólo en disciplinas académicas tales como 
la psicología, sino en el mundo práctico de los negocios. Estos nuevos desarrollos en la construcción de modelos constituyen una de las más excitantes tendencias intelectuales en la época presente, y las extensas aplicaciones de la deducción en estos desarrollos estimula, más bien que reprime, la creatividad humana.

La discusión precedente ha sido destinada a señalar algunos de los contrastes efectivos entre la lógica moderna y la antigua, mostrando al mismo tiempo que algunas diferencias alegadas son puramente imaginarias. También ha intentado poner la lógica deductiva moderna en una perspectiva apropiada mediante la crítica de la tendencia común a confundirla con algunas escuelas y doctrinas filosóficas recientes, y mediante un breve comentario sobre su contribución a la experiencia humana. Más -mucho máscabe decir sobre el último punto, pues fácilmente puede comprobarse que detrás de los tratados aparentemente fríos y puramente intelectuales, existe inequívocamente una cálida actitud humanística.

\section{James Willard Oliver}

(Traducción de Luis O. Hernández León. Revisada por Bernabé Navarro B.) 